\title{
QUASI BOUNDARY TRIPLES AND SEMIBOUNDED SELF-ADJOINT EXTENSIONS
}

\author{
JUSSI BEHRNDT, MATTHIAS LANGER, VLADIMIR LOTOREICHIK, \\ AND JONATHAN ROHLEDER
}

\begin{abstract}
In this note semibounded self-adjoint extensions of symmetric operators are investigated with the help of the abstract notion of quasi boundary triples and their Weyl functions. The main purpose is to provide new sufficient conditions on the parameters in the boundary space to induce self-adjoint realizations, and to relate the decay of the Weyl function to estimates on the lower bound of the spectrum. The abstract results are illustrated with uniformly elliptic second-order PDEs on domains with non-compact boundaries.
\end{abstract}

\section{INTRODUCTION}

Boundary triple techniques are nowadays a widely used abstract tool in extension theory and spectral analysis of symmetric and self-adjoint operators. These methods are inspired by, and can be viewed as, abstract counterparts of trace maps for ordinary or partial differential operators. The Titchmarsh-Weyl $m$-function in singular Sturm-Liouville theory and the Dirichlet-to-Neumann map in the analysis of elliptic differential operators correspond to the Weyl function associated with such a boundary triple. We refer the reader to [12, 13, 21, 40] for ordinary boundary triples, typical applications and further references, to [25, Chapter 13] and $[11,20,22,33,36,37]$ for extension theory of partial differential operators, and to $[4,5,6,7]$ for the more general notion of quasi boundary triples and their use in the spectral analysis of partial differential operators.

The usual starting point is a densely defined, closed, symmetric operator $S$ with equal deficiency indices $n_{ \pm}(S)$ in a Hilbert space $\mathcal{H}$ and its adjoint $S^{*}$. An ordinary boundary triple $\left\{\mathcal{G}, \Gamma_{0}, \Gamma_{1}\right\}$ for $S^{*}$ consists of a Hilbert space $\mathcal{G}$ with $\operatorname{dim} \mathcal{G}=n_{ \pm}(S)$ and two boundary mappings $\Gamma_{0}, \Gamma_{1}: \operatorname{dom} S^{*} \rightarrow \mathcal{G}$ that satisfy an abstract Lagrange or Green identity

$$
\left(S^{*} f, g\right)_{\mathcal{H}}-\left(f, S^{*} g\right)_{\mathcal{H}}=\left(\Gamma_{1} f, \Gamma_{0} g\right)_{\mathcal{G}}-\left(\Gamma_{0} f, \Gamma_{1} g\right)_{\mathcal{G}}, \quad f, g \in \operatorname{dom} S^{*},
$$

and a maximality condition. With the help of a boundary triple the self-adjoint extensions of $S$ in $\mathcal{H}$ can be parameterized in an efficient way via abstract boundary conditions in the boundary space $\mathcal{G}$. More precisely, the restriction

$$
A_{[B]} f:=S^{*} f, \quad \operatorname{dom} A_{[B]}=\left\{f \in \operatorname{dom} S^{*}: \Gamma_{0} f=B \Gamma_{1} f\right\},
$$

of $S^{*}$ is self-adjoint in $\mathcal{H}$ if and only if $B$ is a self-adjoint operator or relation in the boundary space $\mathcal{G}$. The spectral properties of the self-adjoint extensions $A_{[B]}$ can be investigated with the help of the Weyl function $M$ associated with the boundary triple $\left\{\mathcal{G}, \Gamma_{0}, \Gamma_{1}\right\}$. The values $M(\lambda)$ of the Weyl function are linear operators in $\mathcal{G}$ defined by

$$
M(\lambda): \mathcal{G} \rightarrow \mathcal{G}, \quad \Gamma_{0} f \mapsto \Gamma_{1} f, \quad f \in \operatorname{ker}\left(S^{*}-\lambda\right),
$$

2010 Mathematics Subject Classification. Primary 35P15, 47F05; Secondary 35P05, 47B25.

Key words and phrases. semibounded operator, boundary triple, Weyl function, elliptic differential operator, Dirichlet-to-Neumann map. 
where $\lambda \in \mathbb{C}$ does not belong to the spectrum of the self-adjoint extension $A_{0}:=$ $S^{*}\left\lceil\operatorname{ker} \Gamma_{0}\right.$. It turns out that $M$ belongs to the class of operator-valued Nevanlinna or Riesz-Herglotz functions, and, very roughly speaking, the spectral properties of a self-adjoint extension $A_{[B]}$ of $S$ in (1.2) are encoded in the singularities of the function $\lambda \mapsto\left(B^{-1}-M(\lambda)\right)^{-1}$.

For many purposes and applications the notion of boundary triples and their Weyl functions is an efficient and most suitable tool, in particular, for ordinary differential operators and all other extension problems where the deficiency indices of the underlying symmetric operator are finite. However, if one tries to apply the boundary triple method to elliptic PDEs on bounded or unbounded domains with the usual Dirichlet and Neumann trace as boundary maps and the Dirichlet-toNeumann map as Weyl function, one gets into very serious trouble since Green's second identity does not extend to all functions in the maximal domain. There are various ways to overcome this technical difficulty, see $[4,5,11,20,33,36,37,39]$ and the classical contributions $[22,41]$ for more details. One possible solution is the concept of quasi boundary triples, which is a slight generalization of the notion of boundary triples and which was proposed in [4] and further developed and applied in, e.g. $[5,6,7]$. The key idea is to define the boundary maps only on a suitable core of the adjoint operator $S^{*}$ and to require (1.1) to hold only for elements in this core. The notion of the Weyl function in (1.3) remains almost the same: instead of all defect elements $f \in \operatorname{ker}\left(S^{*}-\lambda\right)$, only those belonging to the core are allowed; see Section 2 below. However, it is important to note that for quasi boundary triples some of the striking properties of ordinary boundary triples fail, e.g. self-adjointness of $B$ does not imply, in general, self-adjointness of the extension $A_{[B]}$ in (1.2). Therefore it is desirable to find sufficient conditions for the boundary parameters $B$ to induce self-adjoint extensions $A_{[B]}$ via (1.2). There are some useful sufficient conditions in the literature, most of which rely on compactness properties of the Weyl function; see, e.g. [5, Theorems 6.20 and 6.21] or [4, 6, 7].

One of the main aims of the present paper is to provide new sufficient conditions for the boundary parameter $B$ to induce a self-adjoint extension $A_{[B]}$ via $(1.2)$ in the framework of quasi boundary triples. In Theorems 2.4 and 2.6 and Corollaries 2.5 and 2.7 we drop the above mentioned compactness assumptions and replace them by a set of abstract conditions. In the special but important case when $A_{0}$ is bounded from below and $M(\lambda) \rightarrow 0$ in the operator norm as $\lambda \rightarrow-\infty$, these conditions simplify further; see Theorem 2.8. We emphasize that in the present setting also unbounded self-adjoint operators $B$ are allowed in (1.2).

Our second main objective is to relate decay properties of the Weyl function associated with an ordinary or quasi boundary triple to the lower bounds of the spectra of the self-adjoint extensions $A_{[B]}$. More precisely, since $M$ is a Nevanlinna function, it behaves similarly to the resolvent of the self-adjoint operator $A_{0}$. If $A_{0}$ is bounded from below, the decay of the Weyl function $\lambda \mapsto M(\lambda)$ for $\lambda \rightarrow-\infty$ may be like

$$
\|\overline{M(\lambda)}\|=\mathrm{O}\left(\frac{1}{(\mu-\lambda)^{\alpha}}\right) \quad \text { as } \lambda \rightarrow-\infty,
$$

for some $\alpha \in(0,1]$ and a certain $\mu \leq \min \sigma\left(A_{0}\right)$. This leads to an estimate for the lower bound of the self-adjoint extensions $A_{[B]}$ when the norm of the parameter $B$ tends to $\infty$; see Theorem 2.8 and Corollary 2.9 .

Our general considerations and results in Section 2 are partly inspired by possible applications to elliptic PDEs on unbounded domains with non-compact boundaries. In Section 3 we illustrate our methods with uniformly elliptic second-order differential expressions with smooth variable coefficients. The boundary maps $\Gamma_{0}$ and $\Gamma_{1}$ are chosen to be the Neumann and Dirichlet trace, respectively, defined on $H^{2}(\Omega)$, 
which is a core for the maximal operator. In that case the Weyl function $M$ is the Neumann-to-Dirichlet map, and it is shown in Proposition 3.2 that the norms of the closures $\overline{M(\lambda)}$ satisfy (1.4) with $\alpha=\frac{1}{2}$. As a consequence, the abstract results in Section 2 yield self-adjointness and an estimate for the lower bounds of the spectra of the self-adjoint realizations $A_{[B]}$ in terms of the boundary parameter $B$ in Theorem 3.5. Here the parameter $B$ in the local or non-local Robin boundary condition may also be an unbounded operator. We mention that in certain cases similar estimates can also be obtained via standard techniques involving quadratic forms; see Remark 3.8. Finally, we refer the reader to [18, 19, 26, 27, 28] for a small selection of other recent contributions on spectral properties of elliptic differential operators and especially to $[1,24,32,38,43]$ and the monographs $[23,42]$ for elliptic operators on domains with non-compact boundaries. For further recent contributions to the literature on asymptotics of lower bounds and more explicit spectral asymptotics for elliptic differential operators with Robin boundary conditions we refer the reader to $[17,29,30,31,35]$ and their references.

Acknowledgements. Jussi Behrndt, Vladimir Lotoreichik and Jonathan Rohleder acknowledge financial support by the Austrian Science Fund (FWF): Project P 25162N26. V. L. also acknowledges financial support by the Czech Science Foundation: Project 14-06818S. The authors wish to thank Gerd Grubb for various fruitful discussions and helpful remarks, and, in particular, for pointing out the article [2], which led to the proof of Proposition 3.2 (iv) in its present form.

\section{QuAsi boundary tRIPLES, WEYl FUnCTIONS AND SELF-ADJOINT EXTENSIONS}

Throughout this section we assume that $S$ is a densely defined, closed, symmetric operator in a Hilbert space $\mathcal{H}$. We start by recalling the notion of quasi boundary triples, which was introduced in [4] as a generalization of the concepts of ordinary and generalized boundary triples; for the latter see, e.g. [13, 14].

In the following we denote all appearing inner products by $(\cdot, \cdot)$; the respective Hilbert space will be clear from the context.

Definition 2.1. Let $T \subset S^{*}$ be a linear operator in $\mathcal{H}$ such that $\bar{T}=S^{*}$. A triple $\left\{\mathcal{G}, \Gamma_{0}, \Gamma_{1}\right\}$ is called a quasi boundary triple for $T \subset S^{*}$ if $\mathcal{G}$ is a Hilbert space and $\Gamma_{0}, \Gamma_{1}: \operatorname{dom} T \rightarrow \mathcal{G}$ are linear mappings such that

(i) the abstract Green identity

$$
(T f, g)-(f, T g)=\left(\Gamma_{1} f, \Gamma_{0} g\right)-\left(\Gamma_{0} f, \Gamma_{1} g\right)
$$

holds for all $f, g \in \operatorname{dom} T$;

(ii) the map $\Gamma:=\left(\Gamma_{0}, \Gamma_{1}\right)^{\top}: \operatorname{dom} T \rightarrow \mathcal{G} \times \mathcal{G}$ has dense range;

(iii) $A_{0}:=T \uparrow \operatorname{ker} \Gamma_{0}$ is a self-adjoint operator in $\mathcal{H}$.

We recall from $[4,5]$ that a quasi boundary triple exists if and only if $S$ admits self-adjoint extensions in $\mathcal{H}$, that is, the deficiency indices of $S$ are equal. Moreover, if $\left\{\mathcal{G}, \Gamma_{0}, \Gamma_{1}\right\}$ is a quasi boundary triple for $T \subset S^{*}$, then one has $T=S^{*}$ if and only if $\operatorname{ran} \Gamma=\mathcal{G} \times \mathcal{G}$, in which case $\Gamma=\left(\Gamma_{0}, \Gamma_{1}\right)^{\top}: \operatorname{dom} S^{*} \rightarrow \mathcal{G} \times \mathcal{G}$ is onto and continuous with respect to the graph norm of $S^{*}$, the abstract Green identity holds for all $f, g \in \operatorname{dom} S^{*}$, and the restriction $A_{0}=S^{*} \uparrow \operatorname{ker} \Gamma_{0}$ is automatically self-adjoint. In this situation the notion of quasi boundary triples coincides with the notion of ordinary boundary triples. In particular, this is the case when the deficiency indices of $S$ are finite (and equal). For later use let us also introduce the notation $A_{1}:=T \uparrow \operatorname{ker} \Gamma_{1}$. In contrast to the case of an ordinary boundary triple, this extension of $S$ is not necessarily self-adjoint. 
With each quasi boundary triple $\left\{\mathcal{G}, \Gamma_{0}, \Gamma_{1}\right\}$ one associates a so-called $\gamma$-field and a Weyl function. Before we recall their definitions, note that for each $\lambda \in \rho\left(A_{0}\right)$ one has the direct sum decomposition

$$
\operatorname{dom} T=\operatorname{dom} A_{0} \dot{+} \operatorname{ker}(T-\lambda)=\operatorname{ker} \Gamma_{0} \dot{+} \operatorname{ker}(T-\lambda) .
$$

Thus the restriction of the boundary map $\Gamma_{0}$ to $\operatorname{ker}(T-\lambda)$ is injective, and its range coincides with $\operatorname{ran} \Gamma_{0}$. The definitions of the $\gamma$-field and the Weyl function are now formally the same as for ordinary and generalized boundary triples.

Definition 2.2. The $\gamma$-field $\gamma$ and the Weyl function $M$ corresponding to the quasi boundary triple $\left\{\mathcal{G}, \Gamma_{0}, \Gamma_{1}\right\}$ are defined by

$$
\lambda \mapsto \gamma(\lambda):=\left(\Gamma_{0}\lceil\operatorname{ker}(T-\lambda))^{-1}, \quad \lambda \in \rho\left(A_{0}\right),\right.
$$

and

respectively.

$$
\lambda \mapsto M(\lambda):=\Gamma_{1} \gamma(\lambda), \quad \lambda \in \rho\left(A_{0}\right)
$$

Observe that $\gamma(\lambda)$ is a mapping from $\operatorname{ran} \Gamma_{0} \subset \mathcal{G}$ onto $\operatorname{ker}(T-\lambda) \subset \mathcal{H}$ and that the values $M(\lambda)$ of the Weyl function are operators in $\mathcal{G}$ mapping $\operatorname{ran} \Gamma_{0}$ into $\operatorname{ran} \Gamma_{1}$. Note that $\operatorname{ran} \Gamma_{0}$ and ran $\Gamma_{1}$ are both dense subspaces of $\mathcal{G}$; this is a consequence of the density of the range of $\Gamma=\left(\Gamma_{0}, \Gamma_{1}\right)^{\top}$. Various useful and important properties of the $\gamma$-field and the Weyl function can be found in [4, Proposition 2.6] or [5, Propositions 6.13 and 6.14]. For later purposes we recall that the adjoint $\gamma(\lambda)^{*}$ is a bounded, everywhere defined operator from $\mathcal{H}$ to $\mathcal{G}$, which satisfies

$$
\gamma(\lambda)^{*}=\Gamma_{1}\left(A_{0}-\bar{\lambda}\right)^{-1}, \quad \lambda \in \rho\left(A_{0}\right) .
$$

Furthermore, the values of the Weyl function have the property $M(\lambda) \subset M(\bar{\lambda})^{*}$, $\lambda \in \rho\left(A_{0}\right)$, and, in particular, the operators $M(\lambda)$ are closable. We point out that the operators $M(\lambda)$ and their closures $\overline{M(\lambda)}$ are in general not bounded. However, if $M\left(\lambda_{0}\right)$ is bounded for one $\lambda_{0} \in \rho\left(A_{0}\right)$, then $M(\lambda)$ is bounded for all $\lambda \in \rho\left(A_{0}\right)$; see $[6$, Proposition 3.3 (viii)]. The next lemma, which contains further properties of the Weyl function, is used later.

Lemma 2.3. Let $\left\{\mathcal{G}, \Gamma_{0}, \Gamma_{1}\right\}$ be a quasi boundary triple for $T \subset S^{*}$ with corresponding Weyl function $M$.

(i) For every $\varphi \in\left(\operatorname{ran} \Gamma_{0}\right) \backslash\{0\}$ the function

$$
\lambda \mapsto(M(\lambda) \varphi, \varphi)
$$

is strictly increasing on each interval in $\rho\left(A_{0}\right) \cap \mathbb{R}$.

(ii) If $A_{0}$ is bounded from below and

$$
(M(\lambda) \varphi, \varphi) \rightarrow 0 \quad \text { as } \lambda \rightarrow-\infty
$$

for all $\varphi \in \operatorname{ran} \Gamma_{0}$, then

$$
(M(\lambda) \varphi, \varphi)>0, \quad \varphi \in\left(\operatorname{ran} \Gamma_{0}\right) \backslash\{0\}, \lambda<\min \sigma\left(A_{0}\right) .
$$

Proof. (i) For $\varphi \in\left(\operatorname{ran} \Gamma_{0}\right) \backslash\{0\}$ and $\lambda \in \rho\left(A_{0}\right) \cap \mathbb{R}$ we obtain from [6, Proposition 3.3 (vii)] that

$$
\frac{\mathrm{d}}{\mathrm{d} \lambda}(M(\lambda) \varphi, \varphi)=\left(\gamma(\lambda)^{*} \gamma(\lambda) \varphi, \varphi\right)=\|\gamma(\lambda) \varphi\|^{2}>0
$$

where the last inequality is true since $\gamma(\lambda)$ is injective.

(ii) Relation (2.4) follows directly from (2.3) and (i). 
In contrast to ordinary boundary triples there is no one-to-one correspondence between self-adjoint relations $\Theta$ or $B$ in $\mathcal{G}$ and self-adjoint extensions of $S$ in $\mathcal{H}$ of the form $A_{\Theta}=S^{*} \uparrow \operatorname{ker}\left(\Gamma_{1}-\Theta \Gamma_{0}\right)$ or $A_{[B]}=S^{*} \uparrow \operatorname{ker}\left(B \Gamma_{1}-\Gamma_{0}\right)$, respectively. However, various sufficient conditions for self-adjointness in terms of the parameters $\Theta$ or $B$ were obtained in, e.g. [5, Theorems 6.20 and 6.21] and [6, Theorem 3.11], and, in connection with PDEs on domains with compact boundaries, also in $[4$, Proposition 4.3 and Theorem 4.8] and [6, Theorem 4.5]. In the next theorem we provide a new very useful sufficient condition, which is formulated for the parameter $B=\Theta^{-1}$. In contrast to earlier results no compactness assumption on the values of the Weyl function is imposed. In particular, this allows us to apply the abstract results to elliptic PDEs on domains with non-compact boundaries; see Section 3. We remark that in the application the conditions on $B$ simplify substantially.

Theorem 2.4. Let $\left\{\mathcal{G}, \Gamma_{0}, \Gamma_{1}\right\}$ be a quasi boundary triple for $T \subset S^{*}$ with corresponding $\gamma$-field $\gamma$ and Weyl function $M$. Let $B$ be a linear operator in $\mathcal{G}$ and assume that there exist $\lambda_{ \pm} \in \mathbb{C}^{ \pm}$such that the following conditions are satisfied:

(i) $B$ is symmetric;

(ii) $1 \in \rho\left(B \overline{M\left(\lambda_{ \pm}\right)}\right)$;

(iii) $B\left(\operatorname{ran} \overline{M\left(\lambda_{ \pm}\right)} \cap \operatorname{dom} B\right) \subset \operatorname{ran} \Gamma_{0}$;

(iv) $\operatorname{ran} \Gamma_{1} \subset \operatorname{dom} B$;

(v) $B\left(\operatorname{ran} \Gamma_{1}\right) \subset \operatorname{ran} \Gamma_{0} \quad$ or $A_{1}$ is self-adjoint.

Then the operator

$$
A_{[B]} f=T f, \quad \operatorname{dom} A_{[B]}=\left\{f \in \operatorname{dom} T: \Gamma_{0} f=B \Gamma_{1} f\right\},
$$

is a self-adjoint extension of $S$, and

$$
\left(A_{[B]}-\lambda\right)^{-1}=\left(A_{0}-\lambda\right)^{-1}+\gamma(\lambda)(I-B M(\lambda))^{-1} B \gamma(\bar{\lambda})^{*}
$$

holds for all $\lambda \in \rho\left(A_{[B]}\right) \cap \rho\left(A_{0}\right)$.

Note that if $\left\{\mathcal{G}, \Gamma_{0}, \Gamma_{1}\right\}$ is a generalized boundary triple, i.e. if $\operatorname{ran} \Gamma_{0}=\mathcal{G}$, then (iii) and (v) are automatically satisfied.

Before we prove Theorem 2.4, we state a corollary for bounded $B$, which follows immediately from Theorem 2.4.

Corollary 2.5. Let $\left\{\mathcal{G}, \Gamma_{0}, \Gamma_{1}\right\}$ be a quasi boundary triple for $T \subset S^{*}$ with corresponding $\gamma$-field $\gamma$ and Weyl function $M$. Let $B$ be a bounded self-adjoint operator in $\mathcal{G}$ and assume that there exist $\lambda_{ \pm} \in \mathbb{C}^{ \pm}$such that the following conditions are satisfied:

(i) $1 \in \rho\left(B \overline{M\left(\lambda_{ \pm}\right)}\right)$;

(ii) $B\left(\operatorname{ran} \overline{M\left(\lambda_{ \pm}\right)}\right) \subset \operatorname{ran} \Gamma_{0}$;

(iii) $B\left(\operatorname{ran} \Gamma_{1}\right) \subset \operatorname{ran} \Gamma_{0}$ or $A_{1}$ is self-adjoint.

Then the operator $A_{[B]}$ in (2.5) is a self-adjoint extension of $S$, and the resolvent formula (2.6) holds for all $\lambda \in \rho\left(A_{[B]}\right) \cap \rho\left(A_{0}\right)$.

Proof of Theorem 2.4. The proof of Theorem 2.4 consists of several steps. In the first four steps we assume that the first condition in (v) is satisfied.

Step 1. First we show that $A_{[B]}$ is symmetric, which is essentially a simple consequence of the abstract Green identity (2.1). In fact, by assumption (iv) for $f, g \in \operatorname{dom} A_{[B]}$ we have $\Gamma_{1} f, \Gamma_{1} g \in \operatorname{dom} B$,

$$
B \Gamma_{1} f=\Gamma_{0} f, \quad \text { and } \quad B \Gamma_{1} g=\Gamma_{0} g,
$$


which implies that

$$
\begin{aligned}
\left(A_{[B]} f, g\right)-\left(f, A_{[B]} g\right) & =(T f, g)-(f, T g)=\left(\Gamma_{1} f, \Gamma_{0} g\right)-\left(\Gamma_{0} f, \Gamma_{1} g\right) \\
& =\left(\Gamma_{1} f, B \Gamma_{1} g\right)-\left(B \Gamma_{1} f, \Gamma_{1} g\right)=0,
\end{aligned}
$$

where assumption (i) on the symmetry of the operator $B$ was used in the last step. This shows that $A_{[B]}$ is a symmetric operator in $\mathcal{H}$.

Step 2. In this step we show the inclusions

$$
\operatorname{ran}\left(B \gamma\left(\bar{\lambda}_{ \pm}\right)^{*}\right) \subset \operatorname{ran}\left(I-B M\left(\lambda_{ \pm}\right)\right) .
$$

We consider only $\lambda_{+} \in \mathbb{C}^{+}$; the proof for $\lambda_{-} \in \mathbb{C}^{-}$is the same. Note first that it follows from (2.2) and condition (iv) that the product $B \gamma\left(\bar{\lambda}_{ \pm}\right)^{*}$ is everywhere defined. Let $g \in \operatorname{ran}\left(B \gamma\left(\bar{\lambda}_{+}\right)^{*}\right)$. Then there exists an $f \in \mathcal{H}$ such that $g=$ $B \gamma\left(\bar{\lambda}_{+}\right)^{*} f$. By $(2.2)$ we have $\gamma\left(\bar{\lambda}_{+}\right)^{*} f=\Gamma_{1}\left(A_{0}-\lambda_{+}\right)^{-1} f \in \operatorname{ran} \Gamma_{1}$, and hence assumption (v) implies that

$$
B \gamma\left(\bar{\lambda}_{+}\right)^{*} f \in \operatorname{ran} \Gamma_{0}
$$

We set

$$
\varphi:=\left(I-B \overline{M\left(\lambda_{+}\right)}\right)^{-1} B \gamma\left(\bar{\lambda}_{+}\right)^{*} f
$$

which is well defined by assumption (ii). We can rewrite (2.9) in the form

$$
\varphi=B \overline{M\left(\lambda_{+}\right)} \varphi+B \gamma\left(\bar{\lambda}_{+}\right)^{*} f .
$$

Since $\overline{M\left(\lambda_{+}\right)} \varphi \in \operatorname{ran} \overline{M\left(\lambda_{+}\right)} \cap \operatorname{dom} B$, assumption (iii) and the relations (2.8) and (2.10) imply that $\varphi \in \operatorname{ran} \Gamma_{0}=\operatorname{dom} M\left(\lambda_{+}\right)$. Together with (2.10) this yields

$$
\left(I-B M\left(\lambda_{+}\right)\right) \varphi=B \gamma\left(\bar{\lambda}_{+}\right)^{*} f=g,
$$

and hence $g \in \operatorname{ran}\left(I-B M\left(\lambda_{+}\right)\right)$, i.e. the inclusion (2.7) is shown for $\lambda_{+} \in \mathbb{C}^{+}$.

Step 3. We claim that $\operatorname{ran}\left(A_{[B]}-\lambda_{ \pm}\right)=\mathcal{H}$ holds. Again we show the assertion only for $\lambda_{+} \in \mathbb{C}^{+}$; the arguments for $\lambda_{-} \in \mathbb{C}^{-}$are the same. Let $f \in \mathcal{H}$ and consider the element

$$
h:=\left(A_{0}-\lambda_{+}\right)^{-1} f+\gamma\left(\lambda_{+}\right)\left(I-B M\left(\lambda_{+}\right)\right)^{-1} B \gamma\left(\bar{\lambda}_{+}\right)^{*} f .
$$

Note that by assumption (ii) the inverse $\left(I-B M\left(\lambda_{+}\right)\right)^{-1}$ exists. It maps into $\operatorname{dom} M\left(\lambda_{+}\right)=\operatorname{ran} \Gamma_{0}$, so the product with $\gamma\left(\lambda_{+}\right)$is well defined. Observe also that the product of $\left(I-B M\left(\lambda_{+}\right)\right)^{-1}$ and $B \gamma\left(\bar{\lambda}_{+}\right)^{*}$ is well defined by (2.7). We now show that $h \in \operatorname{dom} A_{[B]}$. Clearly, $h \in \operatorname{dom} T$ since

$$
\left(A_{0}-\lambda_{+}\right)^{-1} f \in \operatorname{dom} A_{0} \subset \operatorname{dom} T
$$

and

$$
\operatorname{ran} \gamma\left(\lambda_{+}\right)=\operatorname{ker}\left(T-\lambda_{+}\right) \subset \operatorname{dom} T .
$$

Furthermore, using (2.2) and the definition of $M\left(\lambda_{+}\right)$we have

$$
\begin{aligned}
B \Gamma_{1} h & =B \Gamma_{1}\left(A_{0}-\lambda_{+}\right)^{-1} f+B \Gamma_{1} \gamma\left(\lambda_{+}\right)\left(I-B M\left(\lambda_{+}\right)\right)^{-1} B \gamma\left(\bar{\lambda}_{+}\right)^{*} f \\
& =B \gamma\left(\bar{\lambda}_{+}\right)^{*} f+B M\left(\lambda_{+}\right)\left(I-B M\left(\lambda_{+}\right)\right)^{-1} B \gamma\left(\bar{\lambda}_{+}\right)^{*} f \\
& =\left[\left(I-B M\left(\lambda_{+}\right)\right)+B M\left(\lambda_{+}\right)\right]\left(I-B M\left(\lambda_{+}\right)\right)^{-1} B \gamma\left(\bar{\lambda}_{+}\right)^{*} f \\
& =\left(I-B M\left(\lambda_{+}\right)\right)^{-1} B \gamma\left(\bar{\lambda}_{+}\right)^{*} f ;
\end{aligned}
$$

the relation $\operatorname{dom} A_{0}=\operatorname{ker} \Gamma_{0}$ and the definition of $\gamma\left(\lambda_{+}\right)$yield

$$
\begin{aligned}
\Gamma_{0} h & =\Gamma_{0}\left(A_{0}-\lambda_{+}\right)^{-1} f+\Gamma_{0} \gamma\left(\lambda_{+}\right)\left(I-B M\left(\lambda_{+}\right)\right)^{-1} B \gamma\left(\bar{\lambda}_{+}\right)^{*} f \\
& =\left(I-B M\left(\lambda_{+}\right)\right)^{-1} B \gamma\left(\bar{\lambda}_{+}\right)^{*} f .
\end{aligned}
$$


Hence the element $h$ in (2.11) satisfies the boundary condition $\Gamma_{0} h=B \Gamma_{1} h$. This shows that $h \in \operatorname{dom} A_{[B]}$. Finally, we obtain from (2.11) that

$$
\left(A_{[B]}-\lambda_{+}\right) h=\left(T-\lambda_{+}\right) h=\left(T-\lambda_{+}\right)\left(A_{0}-\lambda_{+}\right)^{-1} f=f,
$$

where again $\operatorname{ran} \gamma\left(\lambda_{+}\right)=\operatorname{ker}\left(T-\lambda_{+}\right)$was used. Hence $\operatorname{ran}\left(A_{[B]}-\lambda_{+}\right)=\mathcal{H}$ holds.

Step 4. It follows from the symmetry of $A_{[B]}$ shown in Step 1 and the range condition in Step 3 that the operator $A_{[B]}$ is self-adjoint in $\mathcal{H}$. The resolvent formula follows for $\lambda=\lambda_{ \pm}$immediately from the identities (2.11) and (2.12) in Step 3. Assume now that $\lambda \in \rho\left(A_{[B]}\right) \cap \rho\left(A_{0}\right)$ is arbitrary. We claim that the operator $I-B M(\lambda)$ is injective. Indeed, if $\varphi \in \operatorname{ker}(I-B M(\lambda))$ then $\varphi \in \operatorname{dom} M(\lambda)=\operatorname{ran} \Gamma_{0}$ and hence $f:=\gamma(\lambda) \varphi \in \operatorname{ker}(T-\lambda)$, so that $\Gamma_{0} f=\varphi$. From

$$
B \Gamma_{1} f=B M(\lambda) \Gamma_{0} f=B M(\lambda) \varphi=\varphi=\Gamma_{0} f
$$

we conclude that $f \in \operatorname{dom} A_{[B]}$ and hence $f \in \operatorname{ker}\left(A_{[B]}-\lambda\right)$. Since $\lambda \in \rho\left(A_{[B]}\right)$, we obtain $f=0$ and $\varphi=\Gamma_{0} f=0$. Thus $I-B M(\lambda)$ is injective.

Next we show the inclusion

$$
\operatorname{ran}\left(B \gamma(\bar{\lambda})^{*}\right) \subset \operatorname{ran}(I-B M(\lambda)) .
$$

To this end, let $\psi \in \operatorname{ran}\left(B \gamma(\bar{\lambda})^{*}\right)$. Then there exists an $f \in \mathcal{H}$ such that $\psi=$ $B \gamma(\bar{\lambda})^{*} f$. Set

$$
\begin{aligned}
& g:=\left(A_{[B]}-\lambda\right)^{-1} f-\left(A_{0}-\lambda\right)^{-1} f \in \operatorname{ker}(T-\lambda), \\
& k:=\left(A_{[B]}-\lambda\right)^{-1} f \in \operatorname{dom} A_{[B]} .
\end{aligned}
$$

From

$$
\begin{aligned}
& \Gamma_{0} g=\Gamma_{0} k, \\
& \Gamma_{1} g=\Gamma_{1} k-\Gamma_{1}\left(A_{0}-\lambda\right)^{-1} f=\Gamma_{1} k-\gamma(\bar{\lambda})^{*} f
\end{aligned}
$$

we conclude that

$$
(I-B M(\lambda)) \Gamma_{0} k=\Gamma_{0} k-B M(\lambda) \Gamma_{0} g=B \Gamma_{1} k-B \Gamma_{1} g=B \gamma(\bar{\lambda})^{*} f=\psi .
$$

This shows the inclusion in (2.13). Now it follows in exactly the same way as in Step 3 that for $\lambda \in \rho\left(A_{[B]}\right) \cap \rho\left(A_{0}\right)$ the resolvent $\left(A_{[B]}-\lambda\right)^{-1}$ is given by the right-hand side of $(2.6)$.

Step 5. Finally, assume that the second condition in (v) is satisfied, i.e. that $A_{1}$ is self-adjoint. Then $\operatorname{ran} M\left(\lambda_{ \pm}\right)=\operatorname{ran} \Gamma_{1}$ by [4, Proposition 2.6 (iii)]. Hence, if $g \in \operatorname{ran} \Gamma_{1}$ then $g \in \operatorname{dom} B$ by (iv) and $g \in \operatorname{ran} M\left(\lambda_{ \pm}\right) \subset \operatorname{ran}\left(\overline{M\left(\lambda_{ \pm}\right)}\right)$. Now (iii) implies that $B g \in \operatorname{ran} \Gamma_{0}$. This shows that the first condition in (v) is satisfied, and we can apply Steps 1-4 of the proof.

For the case when the spectrum of the self-adjoint operator $A_{0}$ does not cover the whole real line a useful variant of Theorem 2.4 is formulated below. Its proof is almost the same as the proof of Theorem 2.4; here the range condition in Step 3 of the proof needs only to be verified for some real point in $\rho\left(A_{0}\right)$, which then automatically belongs to $\rho\left(A_{[B]}\right)$.

Theorem 2.6. Let $\left\{\mathcal{G}, \Gamma_{0}, \Gamma_{1}\right\}$ be a quasi boundary triple for $T \subset S^{*}$ with corresponding $\gamma$-field $\gamma$ and Weyl function $M$. Let $B$ be a linear operator in $\mathcal{G}$ and assume that there exists a $\lambda_{0} \in \rho\left(A_{0}\right) \cap \mathbb{R}$ such that the following conditions are satisfied:

(i) $B$ is symmetric;

(ii) $1 \in \rho\left(B \overline{M\left(\lambda_{0}\right)}\right)$;

(iii) $B\left(\operatorname{ran} \overline{M\left(\lambda_{0}\right)} \cap \operatorname{dom} B\right) \subset \operatorname{ran} \Gamma_{0}$; 
(iv) $\operatorname{ran} \Gamma_{1} \subset \operatorname{dom} B$;

(v) $B\left(\operatorname{ran} \Gamma_{1}\right) \subset \operatorname{ran} \Gamma_{0} \quad$ or $\quad \lambda_{0} \in \rho\left(A_{1}\right)$.

Then the operator

$$
A_{[B]} f=T f, \quad \operatorname{dom} A_{[B]}=\left\{f \in \operatorname{dom} T: \Gamma_{0} f=B \Gamma_{1} f\right\},
$$

is a self-adjoint extension of $S$ such that $\lambda_{0} \in \rho\left(A_{[B]}\right)$, and

$$
\left(A_{[B]}-\lambda\right)^{-1}=\left(A_{0}-\lambda\right)^{-1}+\gamma(\lambda)(I-B M(\lambda))^{-1} B \gamma(\bar{\lambda})^{*}
$$

holds for all $\lambda \in \rho\left(A_{[B]}\right) \cap \rho\left(A_{0}\right)$.

For completeness the corresponding version of Corollary 2.5 is also stated.

Corollary 2.7. Let $\left\{\mathcal{G}, \Gamma_{0}, \Gamma_{1}\right\}$ be a quasi boundary triple for $T \subset S^{*}$ with corresponding $\gamma$-field $\gamma$ and Weyl function $M$. Let $B$ be a bounded self-adjoint operator in $\mathcal{G}$ and assume that there exists a $\lambda_{0} \in \rho\left(A_{0}\right) \cap \mathbb{R}$ such that the following conditions are satisfied:

(i) $1 \in \rho\left(B \overline{M\left(\lambda_{0}\right)}\right)$;

(ii) $B\left(\operatorname{ran} \overline{M\left(\lambda_{0}\right)}\right) \subset \operatorname{ran} \Gamma_{0}$;

(iii) $B\left(\operatorname{ran} \Gamma_{1}\right) \subset \operatorname{ran} \Gamma_{0} \quad$ or $\quad \lambda_{0} \in \rho\left(A_{1}\right)$.

Then the operator $A_{[B]}$ in (2.14) is a self-adjoint extension of $S$ such that $\lambda_{0} \in$ $\rho\left(A_{[B]}\right)$, and the resolvent formula (2.15) holds for all $\lambda \in \rho\left(A_{[B]}\right) \cap \rho\left(A_{0}\right)$.

In the following theorem we consider the situation that the values of the Weyl function are bounded operators which tend to zero as $\lambda \rightarrow-\infty$. In order to formulate this theorem, let us introduce the following notation. For a self-adjoint operator $B$ with spectral measure $E_{B}(\cdot)$ we define its positive and negative parts by

$$
B_{ \pm}:= \pm \int_{\mathbb{R}_{ \pm}} \lambda \mathrm{d} E_{B}(\lambda)
$$

respectively, so that $B_{ \pm} \geq 0$ and $B=B_{+}-B_{-}$. If $A_{0}$ is bounded from below, the assumption $M(\lambda) \rightarrow 0$ as $\lambda \rightarrow-\infty$ implies that $(M(\lambda) \varphi, \varphi) \rightarrow 0$ as $\lambda \rightarrow-\infty$ for each $\varphi \in \operatorname{ran} \Gamma_{0}$. Recall from Lemma 2.3 that this implies non-negativity of $M(\lambda)$ for $\lambda<\min \sigma\left(A_{0}\right)$; in particular, under these conditions $\overline{M(\lambda)}^{1 / 2}$ is well defined for such $\lambda$.

Theorem 2.8. Let $\left\{\mathcal{G}, \Gamma_{0}, \Gamma_{1}\right\}$ be a quasi boundary triple for $T \subset S^{*}$ with corresponding $\gamma$-field $\gamma$ and Weyl function $M$. Assume that $A_{0}$ is bounded from below and that $M(\lambda)$ is bounded for one (and hence for all) $\lambda \in \rho\left(A_{0}\right)$. Let $B$ be a selfadjoint operator in $\mathcal{G}$ which is bounded from above and assume that the following conditions are satisfied:

(i) $\|\overline{M(\lambda)}\| \rightarrow 0$ as $\lambda \rightarrow-\infty$;

(ii) $\operatorname{ran} \overline{M(\lambda)}^{1 / 2} \subset \operatorname{dom} B$ for all $\lambda<\min \sigma\left(A_{0}\right)$;

(iii) $B(\operatorname{ran} \overline{M(\lambda)}) \subset \operatorname{ran} \Gamma_{0} \quad$ for all $\lambda<\min \sigma\left(A_{0}\right)$;

(iv) $\operatorname{ran} \Gamma_{1} \subset \operatorname{dom} B$;

(v) $B\left(\operatorname{ran} \Gamma_{1}\right) \subset \operatorname{ran} \Gamma_{0} \quad$ or $\quad A_{1}$ is self-adjoint and bounded from below.

Then the operator

$$
A_{[B]} f=T f, \quad \operatorname{dom} A_{[B]}=\left\{f \in \operatorname{dom} T: \Gamma_{0} f=B \Gamma_{1} f\right\},
$$

is a self-adjoint extension of $S$, which is bounded from below, and the resolvent formula (2.15) holds for all $\lambda \in \rho\left(A_{[B]}\right) \cap \rho\left(A_{0}\right)$.

Moreover, the following statements are true. 
(a) If $B \leq 0$, then $\min \sigma\left(A_{[B]}\right) \geq \min \sigma\left(A_{0}\right)$.

(b) If there exist $\alpha \in(0,1], \mu \leq \min \sigma\left(A_{0}\right)$ and $C>0$ such that

$$
\|\overline{M(\lambda)}\| \leq \frac{C}{(\mu-\lambda)^{\alpha}}, \quad \text { for } \lambda<\mu
$$

then the lower bound of $A_{[B]}$ satisfies

$$
\min \sigma\left(A_{[B]}\right) \geq \mu-\left(C\left\|B_{+}\right\|\right)^{1 / \alpha} .
$$

Proof. Assumption (i) and the boundedness of $B_{+}$imply that there exists a $\mu_{0}<$ $\min \sigma\left(A_{0}\right)$ such that

$$
\left\|B_{+} \overline{M(\lambda)}\right\|<1
$$

for all $\lambda \leq \mu_{0}$. In the following let $\lambda \leq \mu_{0}$. Since

$$
\sigma\left(\overline{M(\lambda)}^{1 / 2} B_{+} \overline{M(\lambda)}^{1 / 2}\right) \backslash\{0\}=\sigma\left(B_{+} \overline{M(\lambda)}\right) \backslash\{0\},
$$

relation (2.19) yields that

$$
\sigma\left(\overline{M(\lambda)}^{1 / 2} B_{+} \overline{M(\lambda)}^{1 / 2}\right) \subset[-\beta, \beta]
$$

for some $\beta \in(0,1)$. It follows from assumption (ii), the relation $\operatorname{dom} B_{-}=\operatorname{dom} B$ and the closed graph theorem that

$$
B_{-} \overline{M(\lambda)}^{1 / 2}
$$

is a bounded everywhere defined operator. Hence

$$
\overline{M(\lambda)}^{1 / 2} B_{-} \overline{M(\lambda)}^{1 / 2}
$$

is a bounded, non-negative operator. This, together with (2.20), shows that

$$
\sigma\left(\overline{M(\lambda)}^{1 / 2} B \overline{M(\lambda)}^{1 / 2}\right) \subset(-\infty, \beta]
$$

see, e.g. [9, Lemma 3 in $\S 9.4]$. In particular, we have

$$
1 \in \rho\left(\overline{M(\lambda)}^{1 / 2} B \overline{M(\lambda)}^{1 / 2}\right) .
$$

Since by the closed graph theorem the operator $B \overline{M(\lambda)}^{1 / 2}$ is bounded, it follows from

$$
\sigma\left(\overline{M(\lambda)}^{1 / 2} B \overline{M(\lambda)}^{1 / 2}\right) \backslash\{0\}=\sigma(B \overline{M(\lambda)}) \backslash\{0\},
$$

and (2.21) that $1 \in \rho(B \overline{M(\lambda)})$, i.e. condition (ii) in Theorem 2.6 is satisfied for $\lambda_{0}=\lambda$. Moreover, conditions (ii)-(v) of the current theorem imply conditions (iii)(v) of Theorem 2.6. The latter theorem yields that $A_{[B]}$ is a self-adjoint extension of $S$ and that $\lambda \in \rho\left(A_{[B]}\right)$ for all $\lambda \leq \mu_{0}$, which shows that $A_{[B]}$ is bounded from below.

(a) Assume that $B \leq 0$ and let $\lambda<\min \sigma\left(A_{0}\right)$. Then

$$
\overline{M(\lambda)}^{1 / 2} B \overline{M(\lambda)}^{1 / 2} \leq 0
$$

and hence (2.21) is satisfied. Therefore $1 \in \rho(B \overline{M(\lambda)})$ and, as above, one concludes that $\lambda \in \rho\left(A_{[B]}\right)$. Hence $\min \sigma\left(A_{[B]}\right) \geq \min \sigma\left(A_{0}\right)$.

(b) Now assume that (2.17) is satisfied and let

$$
\lambda<\mu-\left(C\left\|B_{+}\right\|\right)^{1 / \alpha} .
$$

Then

$$
\left\|B_{+} \overline{M(\lambda)}\right\| \leq\left\|B_{+}\right\|\|\overline{M(\lambda)}\|<\frac{(\mu-\lambda)^{\alpha}}{C} \cdot \frac{C}{(\mu-\lambda)^{\alpha}}=1,
$$

i.e. (2.19) is satisfied. The first part of the proof shows that $\lambda \in \rho\left(A_{[B]}\right)$, which proves (2.18). 
Theorem 2.8 implies the following asymptotic estimates on the lower bound of the extensions $A_{[\omega B]}$ of $S$ with $\omega \in \mathbb{R}_{+}$as $\omega \rightarrow+\infty$ and $\omega \rightarrow 0+$.

Corollary 2.9. Let $B$ be a self-adjoint operator in $\mathcal{G}$ which is bounded from above and let the assumptions (i)-(v) and (2.17) from Theorem 2.8 be satisfied. Then the operators

$$
A_{[\omega B]} f=T f, \quad \operatorname{dom} A_{[\omega B]}=\left\{f \in \operatorname{dom} T: \Gamma_{0} f=\omega B \Gamma_{1} f\right\}, \quad \omega \geq 0,
$$

are self-adjoint extensions of $S$, which are bounded from below. Define the function $F(\omega):=\min \sigma\left(A_{0}\right)-\min \sigma\left(A_{[\omega B]}\right)$ and let $F^{+}$be its positive part. Then the following asymptotic estimates are satisfied:

(a) $\left|\min \sigma\left(A_{[\omega B]}\right)\right|=\mathcal{O}\left(|\omega|^{1 / \alpha}\right)$ as $\omega \rightarrow+\infty$;

(b) if the bound (2.17) holds for $\mu=\min \sigma\left(A_{0}\right)$, then $F^{+}(\omega)=\mathcal{O}\left(|\omega|^{1 / \alpha}\right)$ as $\omega \rightarrow 0+$.

Proof. The asymptotic estimate in (a) follows directly from (2.18) and from the fact that $\min \sigma\left(A_{[\omega B]}\right) \leq \min \sigma\left(A_{\mathrm{F}}\right)<\infty$, where $A_{\mathrm{F}}$ is the Friedrichs extension of $S$. The asymptotic estimate in (b) is again a straightforward consequence of (2.18).

\section{Elliptic DifFEREnTIAL OPERATORS ON DOMAINS WITH NON-COMPACT BOUNDARIES}

In this section we apply the abstract results from Section 2 to second-order elliptic differential operators on unbounded domains with non-compact boundaries. We refer the reader to $[4,5]$ and $[6,7]$ for related results for bounded and exterior domains, respectively. Here we shall rely on classical results on the $H^{2}$-regularity of the corresponding Dirichlet and Neumann realizations, and make use of a set of assumptions that can be found in a more general context in [3].

Let $\Omega$ be a domain in $\mathbb{R}^{n}$ which is uniformly regular in the sense of [16, page 72]; see also $[3,10]$. This means that $\partial \Omega$ is $C^{\infty}$-smooth and that there exists a covering of $\Omega$ by open sets $\Omega_{j}, j \in \mathbb{N}$, and $n_{0} \in \mathbb{N}$ such that at most $n_{0}$ of the $\Omega_{j}$ have a non-empty intersection, and a family of $C^{\infty}$-homeomorphisms

$$
\varphi_{j}: \Omega_{j} \cap \Omega \rightarrow \mathcal{B}_{1} \cap\left\{x_{n}>0\right\}, \quad \text { where } \mathcal{B}_{r}=\left\{x \in \mathbb{R}^{n}:\|x\|<r\right\},
$$

such that $\varphi_{j}: \Omega_{j} \cap \partial \Omega \rightarrow \mathcal{B}_{1} \cap\left\{x_{n}=0\right\}$, the derivatives of $\varphi_{j}, j \in \mathbb{N}$, and their inverses are uniformly bounded, and $\bigcup_{j} \varphi_{j}^{-1}\left(\mathcal{B}_{1 / 2}\right)$ covers a uniform neighbourhood of $\partial \Omega$. Note that these assumptions are automatically fulfilled, e.g. for domains with compact $C^{\infty}$-smooth boundaries or for compact, smooth perturbations of half-spaces. Let

$$
\mathcal{L}=-\sum_{j, k=1}^{n} \frac{\partial}{\partial x_{j}} a_{j k} \frac{\partial}{\partial x_{k}}+a
$$

be a differential expression on $\Omega$ with bounded coefficients $a_{j k} \in C^{\infty}(\bar{\Omega})$ satisfying $a_{j k}(x)=\overline{a_{k j}(x)}$ for all $x \in \bar{\Omega}$, and having bounded, uniformly continuous derivatives on $\bar{\Omega}$; cf. [3, (S1)-(S5) in Chapter 4]. Moreover, it is assumed that $a \in L^{\infty}(\Omega)$ is real-valued and that $\mathcal{L}$ is uniformly elliptic, i.e. there exists an $E>0$ such that

$$
\sum_{j, k=1}^{n} a_{j k}(x) \xi_{j} \xi_{k} \geq E \sum_{k=1}^{n} \xi_{k}^{2}, \quad \xi=\left(\xi_{1}, \ldots \xi_{n}\right)^{\top} \in \mathbb{R}^{n}, \quad x \in \bar{\Omega} .
$$

In the following we denote by $H^{s}(\Omega)$ and $H^{s}(\partial \Omega)$ the Sobolev spaces of order $s \geq 0$ on $\Omega$ and $\partial \Omega$, respectively. For $f \in C_{0}^{\infty}(\bar{\Omega}):=\left\{\left.g\right|_{\bar{\Omega}}: g \in C_{0}^{\infty}\left(\mathbb{R}^{n}\right)\right\}$ let

$$
\left.\frac{\partial f}{\partial \nu_{\mathcal{L}}}\right|_{\partial \Omega}:=\left.\sum_{j, k=1}^{n} a_{j k} \nu_{j} \frac{\partial f}{\partial x_{k}}\right|_{\partial \Omega}
$$


be the co-normal derivative of $f$ at $\partial \Omega$ with respect to $\mathcal{L}$, where $\nu=\left(\nu_{1}, \ldots, \nu_{n}\right)^{\top}$ is the unit normal vector field at $\partial \Omega$ pointing outwards. Then Green's identity

$$
(\mathcal{L} f, g)-(f, \mathcal{L} g)=\left(\left.f\right|_{\partial \Omega},\left.\frac{\partial g}{\partial \nu_{\mathcal{L}}}\right|_{\partial \Omega}\right)-\left(\left.\frac{\partial f}{\partial \nu_{\mathcal{L}}}\right|_{\partial \Omega},\left.g\right|_{\partial \Omega}\right)
$$

holds for all $f, g \in C_{0}^{\infty}(\bar{\Omega})$ (see, e.g. [16, Theorem 4.4]); here the inner products on the left-hand side are in $L^{2}(\Omega)$ and the inner products on the right-hand side are in $L^{2}(\partial \Omega)$. Recall that the mapping

$$
f \mapsto\left\{\left.f\right|_{\partial \Omega},\left.\frac{\partial f}{\partial \nu_{\mathcal{L}}}\right|_{\partial \Omega}\right\}, \quad f \in C_{0}^{\infty}(\bar{\Omega}),
$$

extends by continuity to a bounded, surjective map from $H^{2}(\Omega)$ to $H^{3 / 2}(\partial \Omega) \times$ $H^{1 / 2}(\partial \Omega)$, and that Green's identity (3.2) extends to all $f, g \in H^{2}(\Omega)$; see, e.g. [16, Theorem 3.9]. For the extended trace and normal derivative we write again $\left.f\right|_{\partial \Omega}$ and $\left.\frac{\partial f}{\partial \nu_{\mathcal{L}}}\right|_{\partial \Omega}$, respectively.

In order to construct a quasi boundary triple, consider the operators $S$ and $T$ in $L^{2}(\Omega)$ given by

$$
S f=\mathcal{L} f, \quad \operatorname{dom} S=\left\{f \in H^{2}(\Omega):\left.f\right|_{\partial \Omega}=\left.\frac{\partial f}{\partial \nu_{\mathcal{L}}}\right|_{\partial \Omega}=0\right\},
$$

and

$$
T f=\mathcal{L} f, \quad \operatorname{dom} T=H^{2}(\Omega) .
$$

The proof of the following proposition is similar to the proof of [4, Proposition 4.6] and is omitted. We only mention that the self-adjointness of $A_{\mathrm{N}}$ in (ii) follows from [3, Theorem 7.1 (a) and Theorem 7.2].

Proposition 3.1. The operator $S$ in (3.3) is closed, symmetric and densely defined with $\bar{T}=S^{*}$, and the triple $\left\{L^{2}(\partial \Omega), \Gamma_{0}, \Gamma_{1}\right\}$ with

$$
\Gamma_{0} f=\left.\frac{\partial f}{\partial \nu_{\mathcal{L}}}\right|_{\partial \Omega}, \quad \Gamma_{1} f=\left.f\right|_{\partial \Omega}, \quad f \in \operatorname{dom} T,
$$

is a quasi boundary triple for $T \subset S^{*}$ with the following properties:

(i) $\operatorname{ran}\left(\Gamma_{0}, \Gamma_{1}\right)^{\top}=H^{1 / 2}(\partial \Omega) \times H^{3 / 2}(\partial \Omega)$;

(ii) $A_{0}=T \uparrow \operatorname{ker} \Gamma_{0}$ coincides with the self-adjoint Neumann operator

$$
A_{\mathrm{N}} f=\mathcal{L} f, \quad \operatorname{dom} A_{\mathrm{N}}=\left\{f \in H^{2}(\Omega):\left.\frac{\partial f}{\partial \nu_{\mathcal{L}}}\right|_{\partial \Omega}=0\right\},
$$

and $A_{1}=T \uparrow \operatorname{ker} \Gamma_{1}$ coincides with the self-adjoint Dirichlet operator

$$
A_{\mathrm{D}} f=\mathcal{L} f, \quad \operatorname{dom} A_{\mathrm{D}}=\left\{f \in H^{2}(\Omega):\left.f\right|_{\partial \Omega}=0\right\} ;
$$

in particular, $A_{0}$ and $A_{1}$ are bounded from below by essinf $a$.

In the next proposition we prove a couple of properties of the Weyl function, which turns out to be the Neumann-to-Dirichlet map. These properties are needed in order to apply the results from Section 2. In particular, in (iv) we prove a decay estimate for the Weyl function, whose proof is based on an argument due to S. Agmon [2, Section 2]; related methods were also used in the proof of [1, Theorem 4.1]. This estimate can also be shown using the calculus of parameter-dependent pseudodifferential operators provided in, e.g. [23, Chapter 2].

Proposition 3.2. Let $\left\{L^{2}(\partial \Omega), \Gamma_{0}, \Gamma_{1}\right\}$ be the quasi boundary triple from Proposition 3.1 and let $M$ be the corresponding Weyl function. 
(i) The function $M$ is given by the Neumann-to-Dirichlet map, i.e. it satisfies

$$
\left.M(\lambda) \frac{\partial f}{\partial \nu_{\mathcal{L}}}\right|_{\partial \Omega}=\left.f\right|_{\partial \Omega}, \quad f \in \operatorname{ker}(T-\lambda), \quad \lambda \in \rho\left(A_{\mathrm{N}}\right) .
$$

Moreover,

$$
\operatorname{dom} M(\lambda)=H^{1 / 2}(\partial \Omega) \quad \text { and } \quad \operatorname{ran} M(\lambda) \subset H^{3 / 2}(\partial \Omega)
$$

for each $\lambda \in \rho\left(A_{\mathrm{N}}\right)$. If $\lambda<\min \sigma\left(A_{\mathrm{N}}\right)$ then $\operatorname{ran} M(\lambda)=H^{3 / 2}(\partial \Omega)$ and $\operatorname{ker} M(\lambda)=\{0\}$.

(ii) For all $\lambda \in \rho\left(A_{\mathrm{N}}\right)$ the operator $M(\lambda)$ is bounded and non-closed in $L^{2}(\partial \Omega)$, and its closure satisfies $\operatorname{ran} \overline{M(\lambda)} \subset H^{1}(\partial \Omega)$.

(iii) For $\lambda<\min \sigma\left(A_{\mathrm{N}}\right)$ the operator $M(\lambda)$ is non-negative and satisfies

$$
\operatorname{ran} \overline{M(\lambda)}^{1 / 2}=H^{1 / 2}(\partial \Omega)
$$

(iv) For each $\mu<\min \sigma\left(A_{\mathrm{N}}\right)$ there exists a constant $C=C(\mathcal{L}, \Omega, \mu)$ such that

$$
\|\overline{M(\lambda)}\| \leq \frac{C}{(\mu-\lambda)^{1 / 2}}, \quad \lambda<\mu
$$

Proof. (i) The representation of the Weyl function and the assertion (3.5) follow directly from the definition of the boundary maps and the Weyl function. Since $A_{\mathrm{D}}$ is the Friedrichs extension of $S$, each $\lambda<\min \sigma\left(A_{\mathrm{N}}\right)$ belongs to $\rho\left(A_{\mathrm{D}}\right)$. Thus the Dirichlet-to-Neumann map $\left.\left.f\right|_{\partial \Omega} \mapsto \frac{\partial f}{\partial \nu_{\mathcal{L}}}\right|_{\partial \Omega}, f \in \operatorname{ker}(T-\lambda)$, is well defined with domain equal to $H^{3 / 2}(\partial \Omega)$. Since its inverse is given by $M(\lambda)$, we have $\operatorname{ran} M(\lambda)=H^{3 / 2}(\partial \Omega)$ and $\operatorname{ker} M(\lambda)=\{0\}$.

(ii) These properties can be shown in the same way as in [6, Lemma 4.4].

(iii) Let $\mathfrak{a}$ be the quadratic form corresponding to the Neumann operator, i.e.

$$
\begin{aligned}
\mathfrak{a}[f] & :=\mathfrak{a}_{1}[f]+(a f, f) \\
& :=\int_{\Omega}\left(\sum_{j, k=1}^{n} a_{j k} \frac{\partial f}{\partial x_{k}} \cdot \frac{\partial \bar{f}}{\partial x_{j}}\right) \mathrm{d} x+(a f, f), \quad f \in \operatorname{dom} \mathfrak{a}:=H^{1}(\Omega),
\end{aligned}
$$

where $(\cdot, \cdot)$ is the inner product in $L^{2}(\Omega)$. Let $\lambda<\min \sigma\left(A_{\mathrm{N}}\right), \varphi \in H^{1 / 2}(\partial \Omega)$ and let $f \in \operatorname{ker}(T-\lambda)$ such that $\varphi=\left.\frac{\partial f}{\partial \nu_{\mathcal{L}}}\right|_{\partial \Omega}$, i.e. $f=\gamma(\lambda) \varphi$. Then Green's first identity yields

$$
\begin{aligned}
(M(\lambda) \varphi, \varphi) & =\left(\left.f\right|_{\partial \Omega},\left.\frac{\partial f}{\partial \nu_{\mathcal{L}}}\right|_{\partial \Omega}\right)=-(f, \mathcal{L} f)+\mathfrak{a}[f] \\
& \geq\left(-\lambda+\min \sigma\left(A_{\mathrm{N}}\right)\right)\|f\|_{L^{2}(\Omega)}^{2} \geq 0,
\end{aligned}
$$

which shows that $M(\lambda)$ is a non-negative operator.

Next we show (3.6). Let $\lambda<\min \sigma\left(A_{\mathrm{N}}\right)$ and consider the quadratic form in $L^{2}(\partial \Omega)$ defined by

$$
\mathfrak{t}_{\lambda}[\varphi]:=\left(M(\lambda)^{-1} \varphi, \varphi\right), \quad \operatorname{dom} \mathfrak{t}_{\lambda}=H^{3 / 2}(\partial \Omega),
$$

which is well defined by item (i). The form $\mathfrak{t}_{\lambda}$ is densely defined, symmetric and non-negative by (3.9). There exist constants $\widetilde{c}_{1}, \widetilde{c}_{2}>0$ such that

$$
\widetilde{c}_{1}\|f\|_{H^{1}(\Omega)}^{2} \leq \mathfrak{a}[f]-\lambda\|f\|_{L^{2}(\Omega)}^{2} \leq \widetilde{c}_{2}\|f\|_{H^{1}(\Omega)}^{2}, \quad f \in H^{1}(\Omega)
$$


to see the first inequality, set $a_{0}:=\operatorname{essinf} a, \sigma_{0}:=\min \sigma\left(A_{\mathrm{N}}\right)$ and let $\varepsilon>0$. Then (where we write $\|\cdot\|$ for $L^{2}$-norms and use $E$ from (3.1))

$$
\begin{aligned}
\mathfrak{a}[f]-\lambda\|f\|^{2} & =\varepsilon \mathfrak{a}_{1}[f]+\varepsilon(a f, f)+(1-\varepsilon) \mathfrak{a}[f]-\lambda\|f\|^{2} \\
& \geq \varepsilon E\|\nabla f\|^{2}+\varepsilon a_{0}\|f\|^{2}+(1-\varepsilon) \sigma_{0}\|f\|^{2}-\lambda\|f\|^{2} \\
& \geq \min \left\{\varepsilon E, \varepsilon a_{0}+(1-\varepsilon) \sigma_{0}-\lambda\right\}\|f\|_{H^{1}(\Omega)}^{2} .
\end{aligned}
$$

If $\varepsilon$ is small enough, then the last minimum is a positive number. Hence the first inequality in (3.10) is shown. The second inequality follows easily from the boundedness of the coefficients.

For each $\varphi \in H^{3 / 2}(\partial \Omega)$ there exists $f \in \operatorname{ker}(T-\lambda)$ such that $\left.f\right|_{\partial \Omega}=\varphi$. Similarly to $(3.9)$ one obtains

$$
\begin{aligned}
\mathfrak{t}_{\lambda}[\varphi] & =\left(M(\lambda)^{-1} \varphi, \varphi\right)=\left(\left.\frac{\partial f}{\partial \nu_{\mathcal{L}}}\right|_{\partial \Omega},\left.f\right|_{\partial \Omega}\right) \\
& =-(\mathcal{L} f, f)+\mathfrak{a}[f]=\mathfrak{a}[f]-\lambda\|f\|_{L^{2}(\Omega)}^{2}, \quad \varphi \in H^{3 / 2}(\partial \Omega) .
\end{aligned}
$$

Together with (3.10) this yields

$$
\widetilde{c}_{1}\|f\|_{H^{1}(\Omega)}^{2} \leq \mathfrak{t}_{\lambda}[\varphi] \leq \widetilde{c}_{2}\|f\|_{H^{1}(\Omega)}^{2}
$$

for all $\varphi \in H^{3 / 2}(\partial \Omega)$ and corresponding $f \in \operatorname{ker}(T-\lambda)$ with $\left.f\right|_{\partial \Omega}=\varphi$. Since the trace map provides an isomorphism from $\left\{g \in H^{1}(\Omega): \mathcal{L} g=\lambda g\right\}$ onto $H^{1 / 2}(\partial \Omega)$, it follows that there exist $c, C>0$ such that

$$
c\|\varphi\|_{H^{1 / 2}(\partial \Omega)}^{2} \leq \mathfrak{t}_{\lambda}[\varphi] \leq C\|\varphi\|_{H^{1 / 2}(\partial \Omega)}^{2}, \quad \varphi \in H^{3 / 2}(\partial \Omega) .
$$

Hence the domain of the closure of $\mathfrak{t}_{\lambda}$ equals $H^{1 / 2}(\partial \Omega)$. From this we obtain (3.6) because $\overline{M(\lambda)}^{-1}$ is the self-adjoint operator that corresponds to the closure of $\mathfrak{t}_{\lambda}$.

(iv) Let $\mathbb{S}^{1}=\mathbb{R} /(2 \pi \mathbb{Z})$ be the one-dimensional torus and consider the product $\Omega \times \mathbb{S}^{1}$. On this manifold we consider the elliptic differential expression

$$
\mathcal{L}_{\mathbb{S}}=\mathcal{L}-\frac{\partial^{2}}{\partial t^{2}}
$$

where $t$ denotes the variable in $\mathbb{S}^{1}$ and $\mathcal{L}$ acts with respect to the variable $x \in \Omega$. The manifold $\partial \Omega \times \mathbb{S}^{1}$ is the boundary of $\Omega \times \mathbb{S}^{1}$. For $s \geq 0$ let $H^{s}\left(\Omega \times \mathbb{S}^{1}\right)$ and $H^{s}\left(\partial \Omega \times \mathbb{S}^{1}\right)$ be the Sobolev spaces on $\Omega \times \mathbb{S}^{1}$ and $\partial \Omega \times \mathbb{S}^{1}$, respectively. On $H^{2}\left(\Omega \times \mathbb{S}^{1}\right)$ we can define traces $\left.f\right|_{\partial \Omega \times \mathbb{S}^{1}}$ and normal derivatives $\left.\frac{\partial f}{\partial \nu_{\mathcal{L}_{\mathbb{S}}}}\right|_{\partial \Omega \times \mathbb{S}^{1}}$. Note that, for functions of the form $f(x, t)=g(x) h(t)$ with $g \in H^{2}(\Omega)$ and $h \in C^{\infty}\left(\mathbb{S}^{1}\right)$, we have

$$
\left.f\right|_{\partial \Omega \times \mathbb{S}^{1}}=\left.h \cdot g\right|_{\partial \Omega} \quad \text { and }\left.\quad \frac{\partial f}{\partial \nu_{\mathcal{L}_{\mathbb{S}}}}\right|_{\partial \Omega \times \mathbb{S}^{1}}=\left.h \cdot \frac{\partial g}{\partial \nu_{\mathcal{L}}}\right|_{\partial \Omega} .
$$

Next let us introduce the operator

$$
T_{\mathbb{S}} f=\mathcal{L}_{\mathbb{S}} f, \quad \operatorname{dom} T_{\mathbb{S}}=H^{2}\left(\Omega \times \mathbb{S}^{1}\right),
$$

in the space $L^{2}\left(\Omega \times \mathbb{S}^{1}\right)$ and the triple $\left\{L^{2}\left(\partial \Omega \times \mathbb{S}^{1}\right), \Gamma_{0}^{\mathbb{S}}, \Gamma_{1}^{\mathbb{S}}\right\}$ where

$$
\Gamma_{0}^{\mathbb{S}} f=\left.\frac{\partial f}{\partial \nu_{\mathcal{L}_{\mathbb{S}}}}\right|_{\partial \Omega \times \mathbb{S}^{1}} \quad \text { and } \quad \Gamma_{1}^{\mathbb{S}} f=\left.f\right|_{\partial \Omega \times \mathbb{S}^{1}} .
$$

Similarly to Proposition 3.1 one verifies that $\left\{L^{2}\left(\partial \Omega \times \mathbb{S}^{1}\right), \Gamma_{0}^{\mathbb{S}}, \Gamma_{1}^{\mathbb{S}}\right\}$ is a quasi boundary triple for $T_{\mathbb{S}} \subset S_{\mathbb{S}}^{*}$, where $S_{\mathbb{S}}=T_{\mathbb{S}} \uparrow\left(\operatorname{ker} \Gamma_{0}^{\mathbb{S}} \cap \operatorname{ker} \Gamma_{1}^{\mathbb{S}}\right)$ and $A_{\mathrm{N}}^{\mathbb{S}}:=T_{\mathbb{S}}\left\lceil\operatorname{ker} \Gamma_{0}^{\mathbb{S}}\right.$. It follows from (3.11) that for $f(x, t)=g(x) h(t)$ with $g \in H^{2}(\Omega)$ and $h \in C^{\infty}\left(\mathbb{S}^{1}\right)$ we have

$$
\Gamma_{j}^{\mathbb{S}} f=h \Gamma_{j} g, \quad j=0,1 .
$$


By the trace theorem we have

$$
\operatorname{ran} \Gamma_{0}^{\mathbb{S}}=H^{1 / 2}\left(\partial \Omega \times \mathbb{S}^{1}\right) \text { and } \operatorname{ran} \Gamma_{1}^{\mathbb{S}}=H^{3 / 2}\left(\partial \Omega \times \mathbb{S}^{1}\right),
$$

and, as for Proposition 3.1, one shows that the values of the Weyl function $M_{\mathbb{S}}$ corresponding to the quasi boundary triple $\left\{L^{2}\left(\partial \Omega \times \mathbb{S}^{1}\right), \Gamma_{0}^{\mathbb{S}}, \Gamma_{1}^{\mathbb{S}}\right\}$ are bounded (nonclosed) operators in $L^{2}\left(\partial \Omega \times \mathbb{S}^{1}\right)$ with $\operatorname{ran} \overline{M_{\mathbb{S}}(\lambda)} \subset H^{1}\left(\partial \Omega \times \mathbb{S}^{1}\right)$; hence $\overline{M_{\mathbb{S}}(\lambda)}$ can be regarded as a bounded operator from $L^{2}\left(\partial \Omega \times \mathbb{S}^{1}\right)$ to $H^{1}\left(\partial \Omega \times \mathbb{S}^{1}\right)$ for $\lambda \in \rho\left(A_{\mathrm{N}}^{\mathbb{S}}\right)$ by the closed graph theorem.

It is not difficult to see that the operator $A_{\mathrm{N}}^{\mathbb{S}}$ is bounded from below with $\min \sigma\left(A_{\mathrm{N}}^{\mathbb{S}}\right)=\min \sigma\left(A_{\mathrm{N}}\right)$. Let $\mu<\min \sigma\left(A_{\mathrm{N}}\right)$. In the following we consider the case

$$
\lambda<\mu-1<\mu<\lambda_{0}<\min \sigma\left(A_{\mathrm{N}}\right),
$$

where $\lambda_{0}$ is some fixed constant, and we set

$$
m:=\sup _{\lambda \leq \mu-1} \frac{\sqrt{\mu-\lambda}}{\sqrt{\lambda_{0}-\lambda}-1}<\infty .
$$

By the above considerations there exists $C^{\prime}>0$, depending on $\lambda_{0}, \Omega$ and $\mathcal{L}$, with

$$
\left\|M_{\mathbb{S}}\left(\lambda_{0}\right) \psi\right\|_{H^{1}\left(\partial \Omega \times \mathbb{S}^{1}\right)} \leq C^{\prime}\|\psi\|_{L^{2}\left(\partial \Omega \times \mathbb{S}^{1}\right)}, \quad \psi \in H^{1 / 2}\left(\partial \Omega \times \mathbb{S}^{1}\right) .
$$

For an arbitrary $\varphi \in H^{1 / 2}(\partial \Omega)$ and $k \in \mathbb{N}$ let us define

$$
f(x, t):=e^{i k t}\left(\gamma\left(\lambda_{0}-k^{2}\right) \varphi\right)(x), \quad x \in \Omega, t \in \mathbb{S}^{1} .
$$

Then $f \in \operatorname{dom} T_{\mathbb{S}}$ and

$$
\mathcal{L}_{\mathbb{S}} f=\left(\lambda_{0}-k^{2}\right) f+k^{2} f=\lambda_{0} f,
$$

that is, $f \in \operatorname{ker}\left(T_{\mathbb{S}}-\lambda_{0}\right)$. Moreover,

$$
\left(\Gamma_{0}^{\mathbb{S}} f\right)(x, t)=e^{i k t}\left(\Gamma_{0} \gamma\left(\lambda_{0}-k^{2}\right) \varphi\right)(x)=e^{i k t} \varphi(x) .
$$

Hence, setting $\psi_{k, \varphi}(x, t):=e^{i k t} \varphi(x)$ for $t \in \mathbb{S}^{1}$ and $x \in \partial \Omega$ we have

$$
\begin{aligned}
\left(M_{\mathbb{S}}\left(\lambda_{0}\right) \psi_{k, \varphi}\right)(x, t) & =\left(M_{\mathbb{S}}\left(\lambda_{0}\right) \Gamma_{0}^{\mathbb{S}} f\right)(x, t)=\left(\Gamma_{1}^{\mathbb{S}} f\right)(x, t) \\
& =e^{i k t}\left(\Gamma_{1} \gamma\left(\lambda_{0}-k^{2}\right) \varphi\right)(x)=e^{i k t}\left(M\left(\lambda_{0}-k^{2}\right) \varphi\right)(x) .
\end{aligned}
$$

It follows that

$$
\begin{aligned}
\left\|M_{\mathbb{S}}\left(\lambda_{0}\right) \psi_{k, \varphi}\right\|_{H^{1}\left(\partial \Omega \times \mathbb{S}^{1}\right)}^{2} & \geq\left\|\frac{\partial}{\partial t} e^{i k t} M\left(\lambda_{0}-k^{2}\right) \varphi\right\|_{L^{2}\left(\partial \Omega \times \mathbb{S}^{1}\right)}^{2} \\
& =2 \pi k^{2}\left\|M\left(\lambda_{0}-k^{2}\right) \varphi\right\|_{L^{2}(\partial \Omega)}^{2} .
\end{aligned}
$$

Combining this estimate with (3.14) we obtain

$$
\begin{aligned}
\left\|M\left(\lambda_{0}-k^{2}\right) \varphi\right\|_{L^{2}(\partial \Omega)} & \leq \frac{1}{\sqrt{2 \pi} k}\left\|M_{\mathbb{S}}\left(\lambda_{0}\right) \psi_{k, \varphi}\right\|_{H^{1}\left(\partial \Omega \times \mathbb{S}^{1}\right)} \\
& \leq \frac{1}{\sqrt{2 \pi} k} C^{\prime}\left\|\psi_{k, \varphi}\right\|_{L^{2}\left(\partial \Omega \times \mathbb{S}^{1}\right)}=\frac{C^{\prime}}{k}\|\varphi\|_{L^{2}(\partial \Omega)}
\end{aligned}
$$

for all $\varphi \in H^{1 / 2}(\partial \Omega)$ and all $k \in \mathbb{N}$. As $\lambda<\lambda_{0}-1$ by (3.12), there exists $k \in \mathbb{N}$ such that $\lambda_{0}-(k+1)^{2} \leq \lambda<\lambda_{0}-k^{2}$. Since $\lambda \mapsto(M(\lambda) \varphi, \varphi)$ is non-decreasing on $\left(-\infty, \min \sigma\left(A_{\mathrm{N}}\right)\right)$ for every $\varphi \in H^{1 / 2}(\partial \Omega)$ by Lemma 2.3 (i) and $M(\lambda) \geq 0$ by item (ii), also $\lambda \mapsto\|\overline{M(\lambda)}\|$ is non-decreasing on the same interval. Hence

$$
\|\overline{M(\lambda)}\| \leq\left\|\overline{M\left(\lambda_{0}-k^{2}\right)}\right\| \leq \frac{C^{\prime}}{k} \leq \frac{C^{\prime}}{\sqrt{\lambda_{0}-\lambda}-1} \leq \frac{m C^{\prime}}{\sqrt{\mu-\lambda}}
$$


for all $\lambda<\mu-1$, where the constant $m$ in (3.13) was used in the last estimate. It remains to show the estimate in $(3.7)$ for $\lambda \in[\mu-1, \mu)$. Here the monotonicity of $M$ yields

$$
\|\overline{M(\lambda)}\| \leq\|\overline{M(\mu)}\| \leq \frac{1}{\sqrt{\mu-\lambda}}\|\overline{M(\mu)}\|, \quad \lambda \in[\mu-1, \mu),
$$

and hence we have shown (3.7) with $C:=\max \left\{m C^{\prime},\|\overline{M(\mu)}\|\right\}$.

Remark 3.3. A possible choice of the constant $C=C(\mathcal{L}, \Omega, \mu)$ can be read off from the proof of the preceding proposition, namely

$$
C=\max \left\{\sup _{\lambda \leq \mu-1} \frac{\sqrt{\mu-\lambda}}{\sqrt{\lambda_{0}-\lambda}-1}\left\|\overline{M_{\mathbb{S}}\left(\lambda_{0}\right)}\right\|,\|\overline{M(\mu)}\|\right\}
$$

with $\lambda_{0} \in\left(\mu, \min \sigma\left(A_{\mathrm{N}}\right)\right)$, where $M_{\mathbb{S}}\left(\lambda_{0}\right)$ is the Neumann-to-Dirichlet map for the differential expression $\mathcal{L}-\frac{\partial^{2}}{\partial t^{2}}-\lambda_{0}$ on $\Omega \times \mathbb{S}^{1}$, considered as an operator from the space $L^{2}\left(\partial \Omega \times \mathbb{S}^{1}\right)$ to $H^{1}\left(\partial \Omega \times \mathbb{S}^{1}\right)$.

Remark 3.4. In general the assertion of Proposition 3.2 (iv) does not extend to the case $\mu=\min \sigma\left(A_{\mathrm{N}}\right)$. In fact, if $\min \sigma\left(A_{\mathrm{N}}\right)$ is an eigenvalue of $A_{\mathrm{N}}$, then $\min \sigma\left(A_{\mathrm{N}}\right)$ is a (generalized) pole of order one of the analytic (Nevanlinna) function $\lambda \mapsto \overline{M(\lambda)}$ and thus

$$
\|\overline{M(\lambda)}\| \sim \frac{K}{\min \sigma\left(A_{\mathrm{N}}\right)-\lambda} \quad \text { as } \quad \lambda \nearrow \min \sigma\left(A_{\mathrm{N}}\right)
$$

for some $K>0$.

Nevertheless, in some cases the estimate (3.7) holds even for $\mu=\min \sigma\left(A_{\mathrm{N}}\right)$. For instance, in the case of the Laplacian $\mathcal{L}=-\Delta$ on the half-space

$$
\Omega=\mathbb{R}_{+}^{n}=\left\{\left(x^{\prime}, x_{n}\right)^{\top} \in \mathbb{R}^{n}: x^{\prime} \in \mathbb{R}^{n-1}, x_{n}>0\right\}
$$

with boundary $\partial \Omega=\mathbb{R}^{n-1}$ one has $\sigma\left(A_{\mathrm{N}}\right)=[0, \infty)$, and the Neumann-to-Dirichlet map can be calculated explicitly, namely,

$$
\overline{M(\lambda)}=\left(-\Delta_{\mathbb{R}^{n-1}}-\lambda\right)^{-1 / 2}, \quad \lambda<0,
$$

where $-\Delta_{\mathbb{R}^{n-1}}$ is the self-adjoint Laplacian in $L^{2}\left(\mathbb{R}^{n-1}\right)$; see, e.g. [25, (9.65)]. From this representation it follows that

$$
\|\overline{M(\lambda)}\|=\frac{1}{\sqrt{-\lambda}}, \quad \lambda<0 .
$$

We remark that the asymptotic behaviour of the Neumann-to-Dirichlet map for the Laplacian on a half-space was also used in the context of spectral theory in, e.g. [32].

The following theorem shows the self-adjointness of elliptic differential operators with generalized Robin boundary conditions and yields a bound for the minima of their spectra. Note that the $\gamma$-field corresponding to the quasi boundary triple in Proposition 3.1 is the mapping $\varphi \mapsto \gamma(\lambda) \varphi=f$, where $f \in \operatorname{dom} T$ is the unique solution of the boundary value problem $\mathcal{L} f=\lambda f, \Gamma_{0} f=\left.\frac{\partial f}{\partial \nu_{\mathcal{L}}}\right|_{\partial \Omega}=\varphi \in H^{1 / 2}(\partial \Omega)$.

Theorem 3.5. Let $B$ be a self-adjoint operator in $L^{2}(\partial \Omega)$ which is bounded from above and assume that $H^{1 / 2}(\partial \Omega) \subset \operatorname{dom} B$ and $B\left(H^{1}(\partial \Omega)\right) \subset H^{1 / 2}(\partial \Omega)$. Then the operator

$$
A_{[B]} f=\mathcal{L} f, \quad \operatorname{dom} A_{[B]}=\left\{f \in H^{2}(\Omega):\left.\frac{\partial f}{\partial \nu_{\mathcal{L}}}\right|_{\partial \Omega}=\left.B f\right|_{\partial \Omega}\right\},
$$

is self-adjoint in $L^{2}(\Omega)$ and

$$
\left(A_{[B]}-\lambda\right)^{-1}=\left(A_{\mathrm{N}}-\lambda\right)^{-1}+\gamma(\lambda)(I-B M(\lambda))^{-1} B \gamma(\bar{\lambda})^{*}
$$


holds for all $\lambda \in \rho\left(A_{[B]}\right) \cap \rho\left(A_{\mathrm{N}}\right)$, where $\gamma$ is the $\gamma$-field corresponding to the quasi boundary triple in Proposition 3.1 and $M$ is the Neumann-to-Dirichlet map. Moreover, the self-adjoint operator $A_{[B]}$ is bounded from below with lower bound satisfying

$$
\min \sigma\left(A_{[B]}\right) \geq \mu-\left(C\left\|B_{+}\right\|\right)^{2}
$$

for each $\mu<\min \sigma\left(A_{\mathrm{N}}\right)$, where $C=C(\Omega, \mathcal{L}, \mu)$ is given in Remark 3.3 and $B_{+}$is the positive part of $B$; see (2.16). Moreover, if $B \leq 0$ then $\min \sigma\left(A_{[B]}\right) \geq \min \sigma\left(A_{\mathrm{N}}\right)$.

Proof. Propositions 3.1 and 3.2 show that all assumptions of Theorem 2.8 are satisfied. Hence the latter yields all assertions of the current theorem.

Remark 3.6. The boundary conditions discussed in Theorem 3.5 contain classical Robin boundary conditions. Here one chooses $B f=b f, f \in L^{2}(\partial \Omega)$, for some suitable function $b: \partial \Omega \rightarrow \mathbb{R}$ satisfying the assumptions in the theorem; in this case

$$
B_{+} f=b_{+} f, \quad f \in \operatorname{dom} B,
$$

where $b_{+}$is the positive part of the function $b$. Note also that not every Robin boundary condition with a real valued function $b$ leads to a self-adjoint realization. An example with an unbounded $b$ and its physical motivation were discussed in $[15$, Section 3]; see also [8, 34] for related problems.

Let us formulate a consequence of the previous theorem: under the assumptions of Theorem 3.5 the operators

$$
A_{[\omega B]} f=\mathcal{L} f, \quad \operatorname{dom} A_{[\omega B]}=\left\{f \in H^{2}(\Omega):\left.\frac{\partial f}{\partial \nu_{\mathcal{L}}}\right|_{\partial \Omega}=\left.\omega B f\right|_{\partial \Omega}\right\},
$$

with $\omega \geq 0$ are self-adjoint in $L^{2}(\Omega)$ and bounded from below, and as in Corollary 2.9 (a) one can derive the following asymptotic estimate for the lower bound of $\sigma\left(A_{[\omega B]}\right)$ when the coupling constant $\omega$ tends to $+\infty$.

Corollary 3.7. Let the assumptions of Theorem 3.5 be satisfied. Then

$$
\left|\min \sigma\left(A_{[\omega B]}\right)\right|=\mathcal{O}\left(|\omega|^{2}\right) \text { as } \omega \rightarrow+\infty .
$$

Remark 3.8. We point out that the operator $A_{[B]}$ in Theorem 3.5 can also be defined as the self-adjoint operator representing the closed, densely defined, symmetric and lower-semibounded sesquilinear form

$$
\mathfrak{a}_{B}[f, g]:=\mathfrak{a}[f, g]-\left(\left.B f\right|_{\partial \Omega},\left.g\right|_{\partial \Omega}\right), \quad \operatorname{dom} \mathfrak{a}_{B}:=H^{1}(\Omega),
$$

where $\mathfrak{a}$ is defined as in $(3.8)$ and $(\cdot, \cdot)$ denotes the inner product in $L^{2}(\partial \Omega)$. With this approach additional arguments are needed to show $H^{2}$-regularity of the functions in $\operatorname{dom} A_{[B]}$. Note that the decomposition $B=B_{+}-B_{-}$yields the estimate

$$
\mathfrak{a}_{B}[f] \geq \mathfrak{a}[f]-\left(\left.B_{+} f\right|_{\partial \Omega},\left.f\right|_{\partial \Omega}\right), \quad f \in H^{1}(\Omega) .
$$

Moreover, for any $\varepsilon>0$ there exists a constant $\beta(\varepsilon)>0$ such that

$$
\left\|\left.f\right|_{\partial \Omega}\right\|^{2} \leq \varepsilon\|\nabla f\|^{2}+\beta(\varepsilon)\|f\|^{2}, \quad f \in H^{1}(\Omega) ;
$$

see, e.g. [16, Lemma 3.1]. According to this estimate we have

$$
\left(\left.B_{+} f\right|_{\partial \Omega},\left.f\right|_{\partial \Omega}\right)_{\partial \Omega} \leq\left\|B_{+}\right\|\left\|\left.f\right|_{\partial \Omega}\right\|^{2} \leq \varepsilon\left\|B_{+}\right\|\|\nabla f\|^{2}+\beta(\varepsilon)\left\|B_{+}\right\|\|f\|^{2}
$$

for all $f \in H^{1}(\Omega)$. The ellipticity of $\mathcal{L}$ yields $E\|\nabla f\|^{2} \leq \mathfrak{a}_{1}[f]$ for all $f \in H^{1}(\Omega)$, where $E$ is chosen as in (3.1). Thus, if $B_{+} \neq 0$, then for $\varepsilon=\frac{E}{\left\|B_{+}\right\|}$and any $f \in H^{1}(\Omega)$ we obtain the relation

$$
\begin{aligned}
\mathfrak{a}_{B}[f] & \geq E\|\nabla f\|^{2}+\operatorname{ess} \inf a\|f\|^{2}-E\|\nabla f\|^{2}-\beta\left(\frac{E}{\left\|B_{+}\right\|}\right)\left\|B_{+}\right\|\|f\|^{2} \\
& =\left(\operatorname{essinf} a-\beta\left(\frac{E}{\left\|B_{+}\right\|}\right)\left\|B_{+}\right\|\right)\|f\|^{2},
\end{aligned}
$$


where we used (3.15). In particular,

$$
\min \sigma\left(A_{[B]}\right) \geq \operatorname{ess} \inf a-\beta\left(\frac{E}{\left\|B_{+}\right\|}\right)\left\|B_{+}\right\| .
$$

The possible choice of the constant $\beta(\varepsilon)$ depends on the domain $\Omega$ and has been investigated for certain classes of domains. For instance, if $\Omega$ is a bounded domain (with Lipschitz boundary), then one can choose $\beta$ such that $\beta(\varepsilon)=c / \varepsilon$ for sufficiently small $\varepsilon$ and some $c>0$; see [18, Lemma 2.5]. In this case (3.16) reads

$$
\min \sigma\left(A_{[B]}\right) \geq \operatorname{essinf} a-\frac{c}{E}\left\|B_{+}\right\|^{2}
$$

when $\left\|B_{+}\right\|$is sufficiently large.

\section{REFERENCES}

[1] H. Abels, G. Grubb and I. G. Wood, Extension theory and Kreĭn-type resolvent formulas for nonsmooth boundary value problems, J. Funct. Anal. 266 (2014), 4037-4100.

[2] S. Agmon, On the eigenfunctions and on the eigenvalues of general elliptic boundary value problems, Commun. Pure Appl. Math. 15 (1962), 119-147.

[3] R. Beals, Non-local boundary value problems for elliptic operators, Amer. J. Math. 87 (1965), 315-362.

[4] J. Behrndt and M. Langer, Boundary value problems for elliptic partial differential operators on bounded domains, J. Funct. Anal. 243 (2007), 536-565.

[5] J. Behrndt and M. Langer, Elliptic operators, Dirichlet-to-Neumann maps and quasi boundary triples, in: Operator Methods for Boundary Value Problems, London Math. Soc. Lecture Note Series, vol. 404, 2012, pp. 121-160.

[6] J. Behrndt, M. Langer and V. Lotoreichik, Spectral estimates for resolvent differences of self-adjoint elliptic operators, Integral Equations Operator Theory 77 (2013), 1-37.

[7] J. Behrndt and T. Micheler, Elliptic differential operators on Lipschitz domains and abstract boundary value problems, J. Funct. Anal. 267 (2014), 3657-3709.

[8] M. V. Berry, Hermitian boundary conditions at a Dirichlet singularity: the MarlettaRozenblum model, J. Phys. A 42 (2009), 165208, 13 pp.

[9] M.Sh. Birman and M.Z. Solomjak, Spectral Theory of Self-Adjoint Operators in Hilbert Spaces, D. Reidel Publishing Co., Dordrecht, 1987.

[10] F.E. Browder, On the spectral theory of elliptic differential operators. I, Math. Ann. 142 (1961), 22-130.

[11] B. M. Brown, G. Grubb and I. G. Wood, $M$-functions for closed extensions of adjoint pairs of operators with applications to elliptic boundary problems, Math. Nachr. 282 (2009), 314-347.

[12] J. Brüning, V. Geyler and K. Pankrashkin, Spectra of self-adjoint extensions and applications to solvable Schrödinger operators, Rev. Math. Phys. 20 (2008), 1-70.

[13] V.A. Derkach and M. M. Malamud, Generalized resolvents and the boundary value problems for Hermitian operators with gaps, J. Funct. Anal. 95 (1991), 1-95.

[14] V.A. Derkach and M. M. Malamud, The extension theory of Hermitian operators and the moment problem, J. Math. Sci. 73 (1995), 141-242.

[15] P. Exner and P. Šeba, A simple model of thin-film point contact in two and three dimensions, Czechoslovak J. Phys. B 38 (1988), 1095-1110.

[16] R. Freeman, Closed operators and their adjoints associated with elliptic differential operators, Pacific J. Math. 22 (1967), 71-97.

[17] P. Freitas and D. Krejcirik, The first Robin eigenvalue with negative boundary parameter, Adv. Math. 280 (2015), 322-339.

[18] F. Gesztesy and M. Mitrea, Generalized Robin boundary conditions, Robin-to-Dirichlet maps, and Krein-type resolvent formulas for Schrödinger operators on bounded Lipschitz domains, in: Perspectives in Partial Differential Equations, Harmonic Analysis and Applications, Proc. Sympos. Pure Math., vol. 79, Amer. Math. Soc., Providence, RI, 2008, pp. 105-173.

[19] F. Gesztesy and M. Mitrea, Generalized Robin Laplacians and some remarks on a paper by Filonov on eigenvalue inequalities, J. Differential Equations 247 (2009), 2871-2896.

[20] F. Gesztesy and M. Mitrea, A description of all self-adjoint extensions of the Laplacian and Krel̆n-type resolvent formulas on non-smooth domains, J. Anal. Math. 113 (2011), 53-172.

[21] V.I. Gorbachuk and M. L. Gorbachuk, Boundary Value Problems for Operator Differential Equations, Kluwer Academic Publ., Dordrecht, 1991.

[22] G. Grubb, A characterization of the non-local boundary value problems associated with an elliptic operator, Ann. Scuola Norm. Sup. Pisa (3) 22 (1968), 425-513. 
[23] G. Grubb, Functional Calculus of Pseudodifferential Boundary Problems, Progress in Mathematics, 65, Birkhäuser Boston, MA, 1996.

[24] G. Grubb, Krein resolvent formulas for elliptic boundary problems in nonsmooth domains, Rend. Semin. Mat. Univ. Politec. Torino 66 (2008), 271-297.

[25] G. Grubb, Distributions and Operators. Graduate Texts in Mathematics, vol. 252, Springer, New York, 2009.

[26] G. Grubb, The mixed boundary value problem, Krein resolvent formulas and spectral asymptotic estimates, J. Math. Anal. Appl. 382 (2011), 339-363.

[27] G. Grubb, Extension theory for elliptic partial differential operators with pseudodifferential methods, in: Operator Methods for Boundary Value Problems, London Math. Soc. Lecture Note Series, vol. 404, 2012, pp. 221-258.

[28] G. Grubb, Krein-like extensions and the lower boundedness problem for elliptic operators, J. Differential Equations 252 (2012), 852-885.

[29] B. Helffer and A. Kachmar, Eigenvalues for the Robin Laplacian in domains with variable curvature, Trans. Amer. Math. Soc. 369 (2017), 3253-3287.

[30] B. Helffer and K. Pankrashkin, Tunneling between corners for Robin Laplacians, J. London Math. Soc. (2) 91 (2015) 225-248.

[31] M. Levitin and L. Parnovski, On the principal eigenvalue of a Robin problem with a large parameter, Math. Nachr. 281 (2008), 272-281.

[32] V. Lotoreichik and J. Rohleder, Schatten-von Neumann estimates for resolvent differences of Robin Laplacians on a half-space, Oper. Theory Adv. Appl. 221 (2012), 453-468.

[33] M. M. Malamud, Spectral theory of elliptic operators in exterior domains, Russ. J. Math. Phys. 17 (2010), 96-125.

[34] M. Marletta and G. Rozenblum, A Laplace operator with boundary conditions singular at one point. J. Phys. A 42 (2009), 125204, 11 pp.

[35] K. Pankrashkin and N. Popoff, An effective Hamiltonian for the eigenvalue asymptotics of a Robin Laplacian with a large parameter, J. Math. Pures Appl. 106 (2016), 615-650.

[36] A. Posilicano, Self-adjoint extensions of restrictions, Oper. Matrices 2 (2008), 1-24.

[37] O. Post, Boundary pairs associated with quadratic forms, Math. Nachr. 289 (2016),10521099.

[38] V. Rabinovich, Boundary problems for domains with conical exits at infinity and limit operators, Complex Var. Elliptic Equ. 60 (2015), 293-309.

[39] V. Ryzhov, A general boundary value problem and its Weyl function, Opuscula Math. 27 (2007), 305-331.

[40] K. Schmüdgen, Unbounded Self-adjoint Operators on Hilbert Space, Graduate Texts in Mathematics, vol. 265, Springer, Dordrecht, 2012.

[41] M. I. Vishik, On general boundary problems for elliptic differential equations, Trudy Moskov. Mat. Obšč. 1 (1952), 187-246 (in Russian).

[42] V. Volpert, Elliptic Partial Differential Equations. Volume 1: Fredholm Theory of Elliptic Problems in Unbounded Domains, Monographs in Mathematics, vol. 101, Birkhäuser, Basel, 2011.

[43] A. Volpert and V. Volpert, The Fredholm property of elliptic operators in unbounded domains, Tr. Mosk. Mat. Obs. 67 (2006), 148-227 (in Russian); translation in Trans. Moscow Math. Soc. 67 (2006), 127-197.

Technische Universität Graz, Institut für Numerische Mathematik, Steyrergasse 30, 8010 Graz, Austria

E-mail address: behrndt@tugraz.at

Department of Mathematics and Statistics, University of Strathclyde, 26 Richmond Street, Glasgow G1 1XH, United Kingdom

E-mail address: m.langer@strath.ac.uk

Department of Theoretical Physics, Nuclear Physics Institute ASCR, 25068 Řež near Prague, Czech Republic

E-mail address: lotoreichik@ujf.cas.cz

Stockholms universitet, Matematik, 10691 Stockholm, Sweden

E-mail address: jonathan.rohleder@math.su.se 